\title{
Improving BEL1D accuracy for geophysical imaging of the subsurface
}

Hadrien MICHEL ${ }^{(1,2,3)}$, Thomas HERMANS ${ }^{(2)}$, Thomas KREMER ${ }^{(4)}$, Frédéric NGUYEN(1)

(1) ULiège - Liège, Belgium

(2) UGent - Gent, Belgium

(3) F.R.S.-FNRS - Brussels, Belgium

(4) UNantes - Nantes, France

$\underline{\underline{\underline{\text { IIIIIII }}}}$ GHENT UNIVERSITY

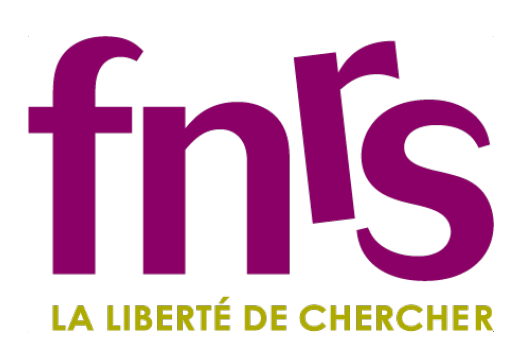

LA LIBERTÉ DE CHERCHER
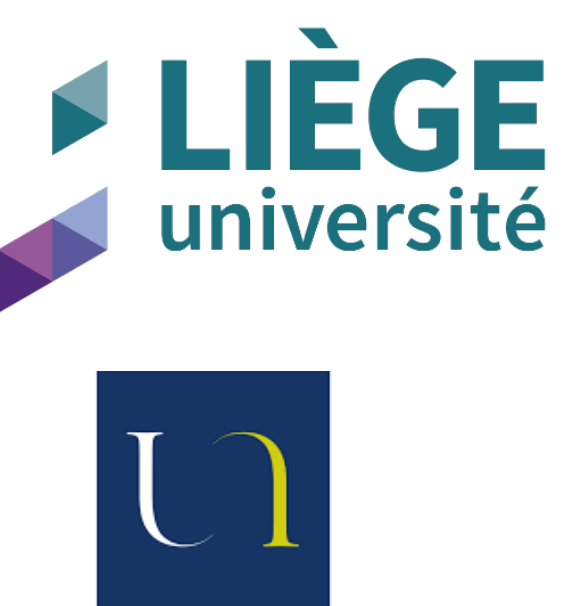

UNIVERSITÉ DE NANTES 


\section{Table of content}

- Basics:

- sNMR - detecting water from the surface

- BEL1D (Michel et al., 2020, Computers \& Geoscience)

- Numerical benchmark:

- Applying BEL1D

- Improving with Iterative Prior Resampling (IPR)

- Comparison with McMC

- Case study: Mont Rigi

- Conclusion and perspectives 


\section{sNMR}

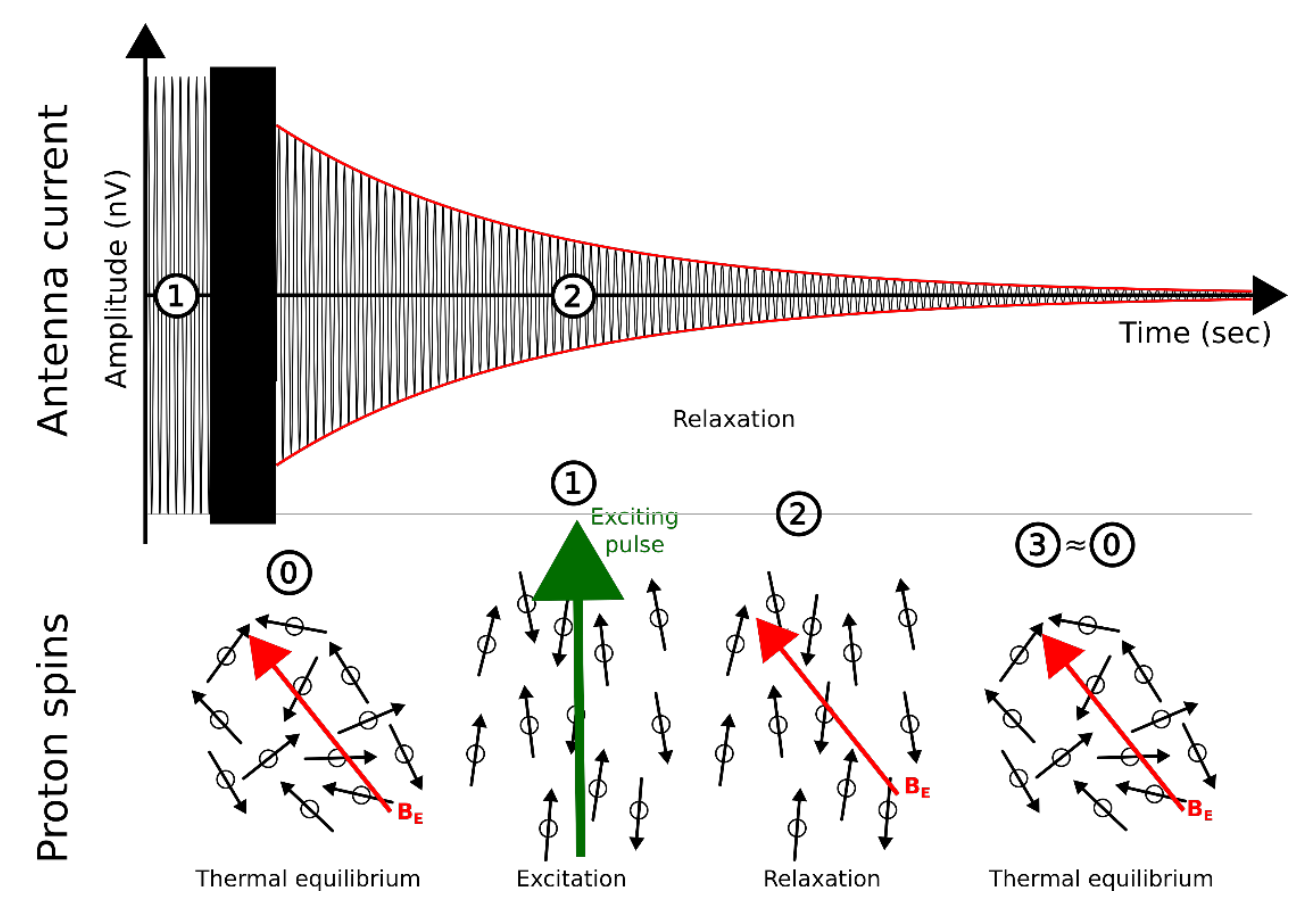

- $s N M R=$ surface Nuclear Magnetic Resonance

- Detecting groundwater from the surface

- After inversion:

- Water content distribution

- Relaxation time distribution 


\section{BEL1D}

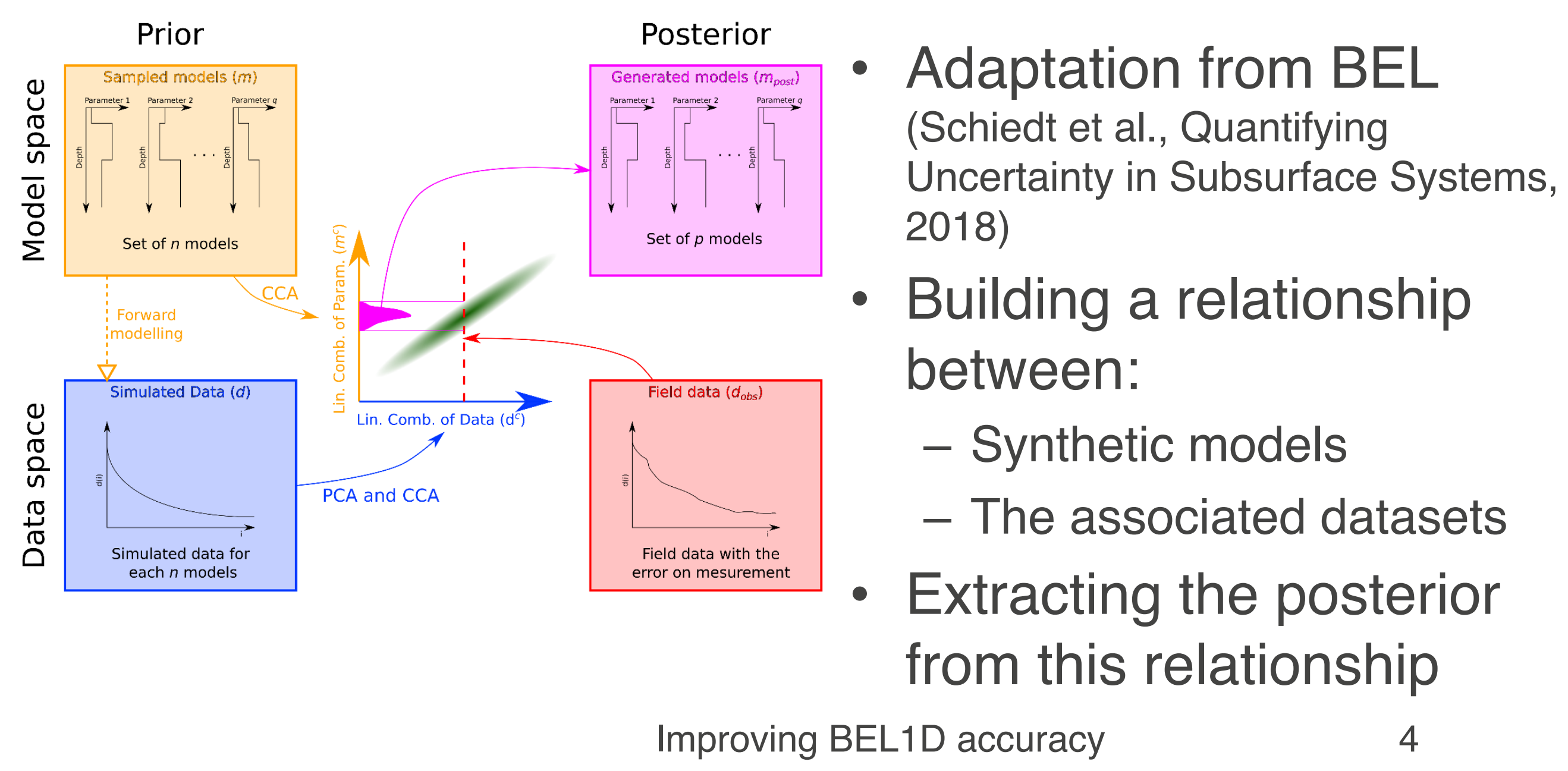




\section{BEL1D}

\section{Sampling models and forward modelling}
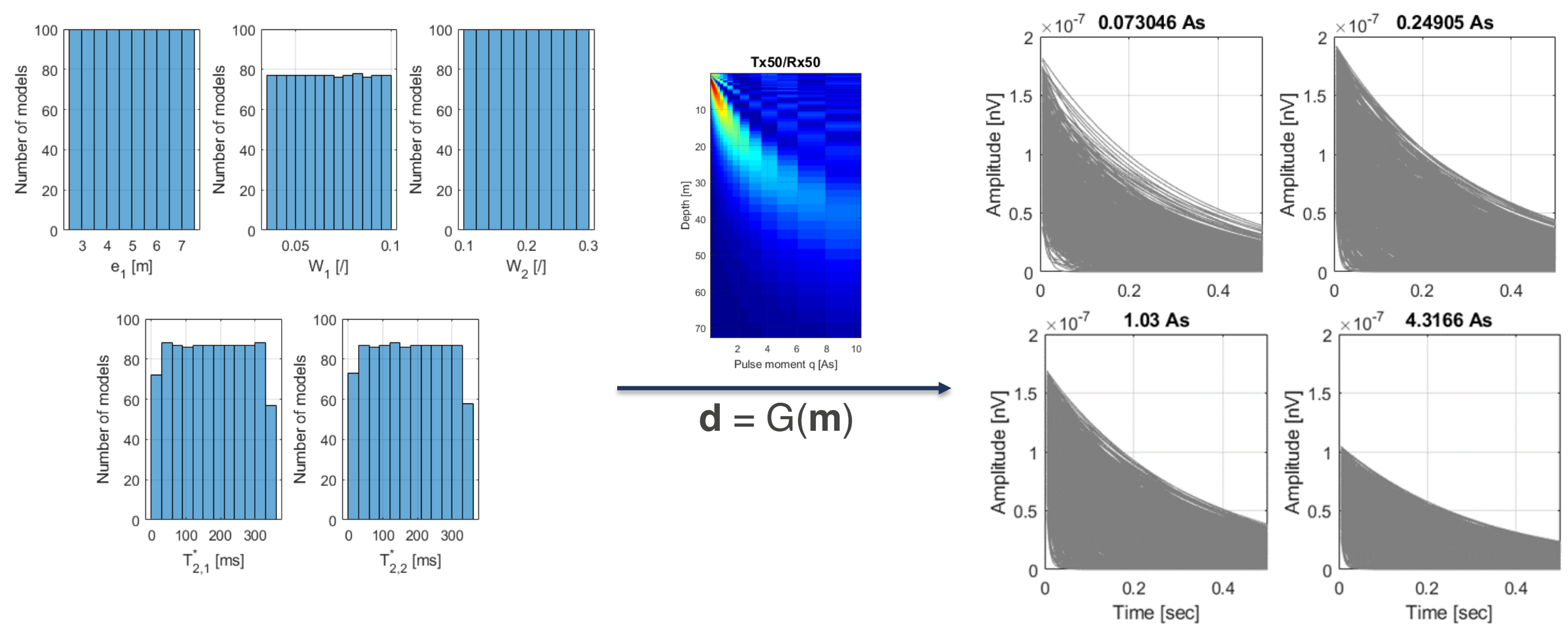

Improving BEL1D accuracy 


\section{BEL1D \\ Reducing dimensionality (PCA)}

- From 10,000 dimensions in the dataset to around 10

- Keeping $90 \%$ variability

- Not applied to the models

- Uncorrelated prior

- Poor performances

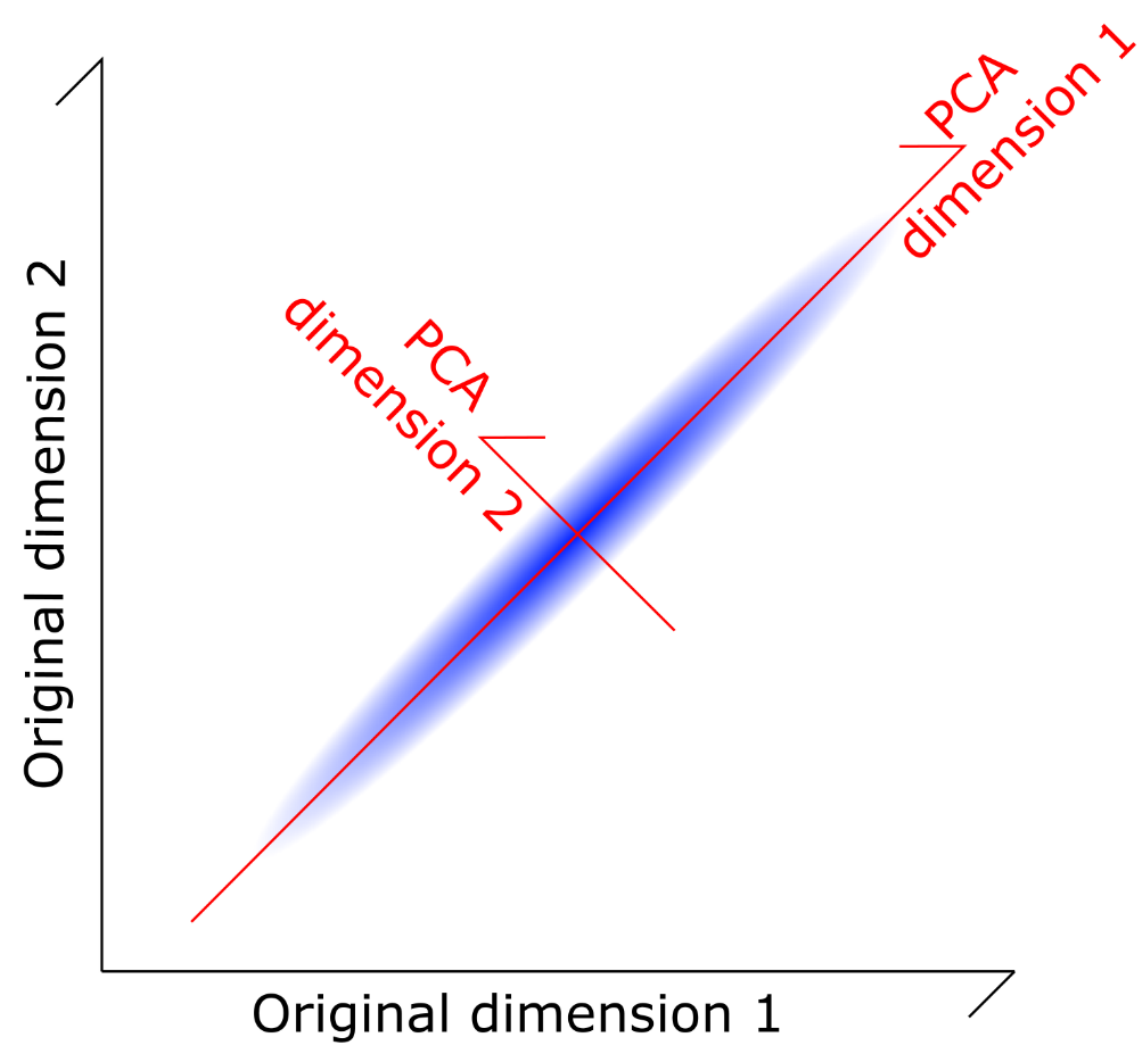




\section{BEL1D}

Canonical correlation analysis

- Linking the models parameters to the reduced datasets $\rightarrow$ CCA
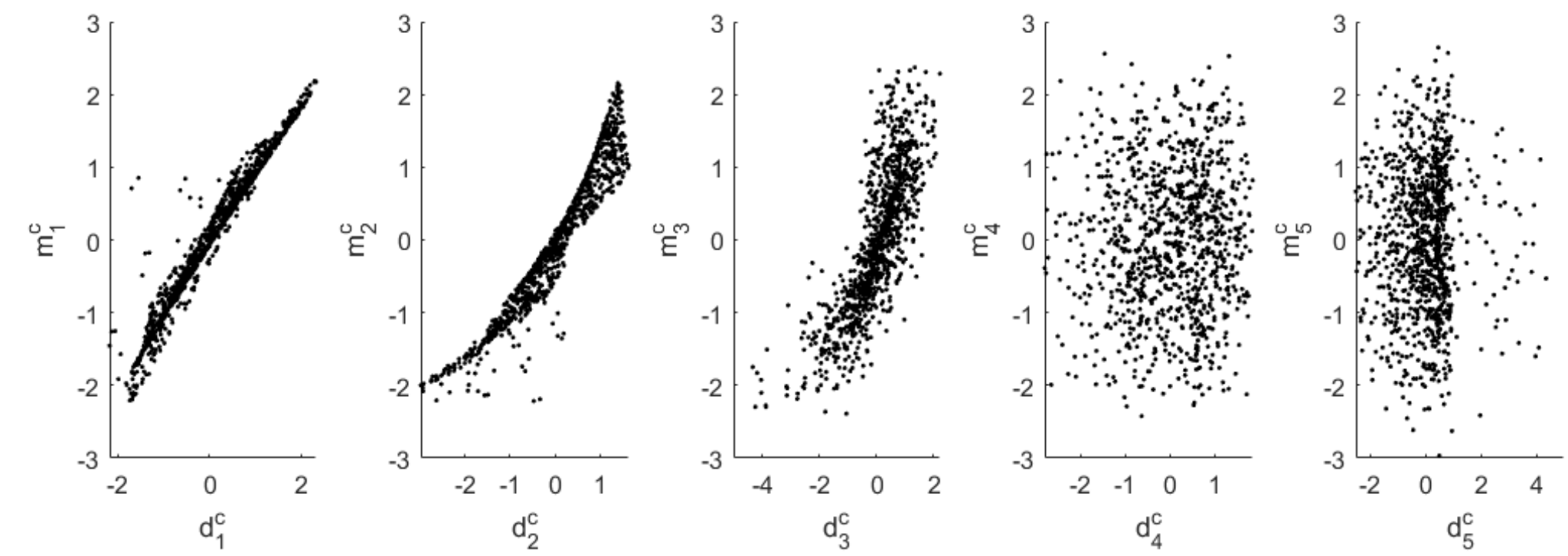

Improving BEL1D accuracy 


\section{BEL1D}

Extracting the posterior in reduced space
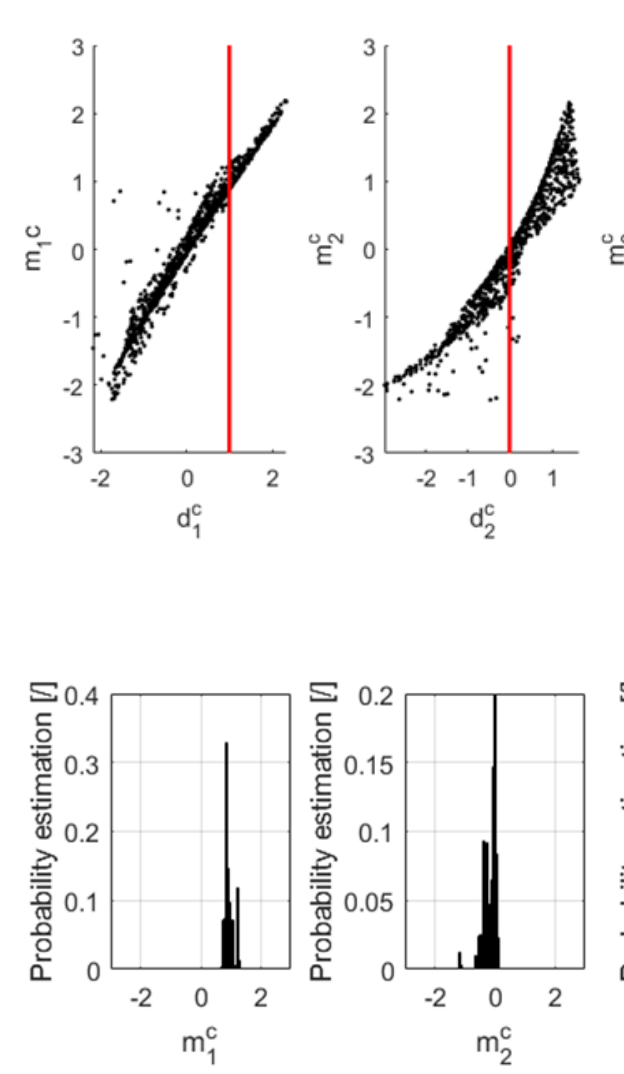
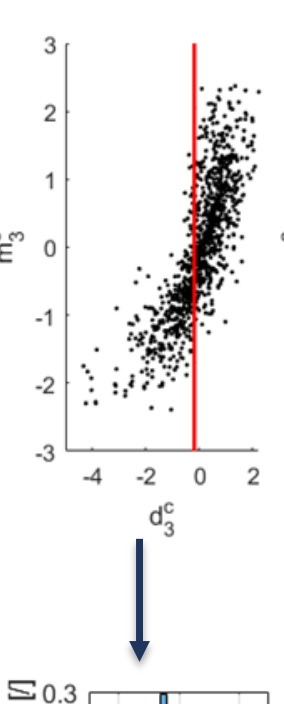

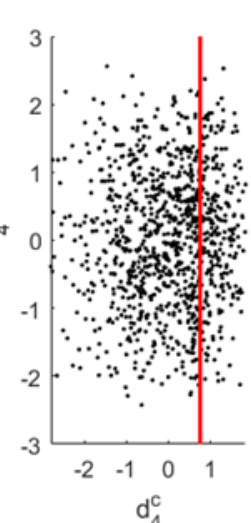

$d_{4}^{c}$

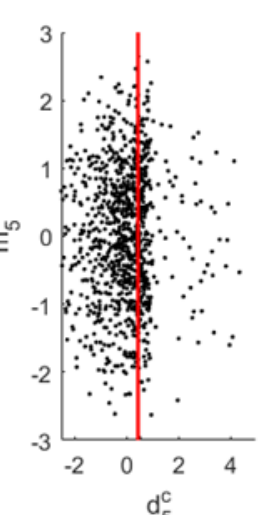

- Transform the field dataset (PCA and CCA)

- Report in the CCA space

- Extract the obtained distribution (Kernel Density Estimation) 


\section{BEL1D \\ Back-transform into original space}

- Apply the inverse transform (CCA) to sampled models in reduced space

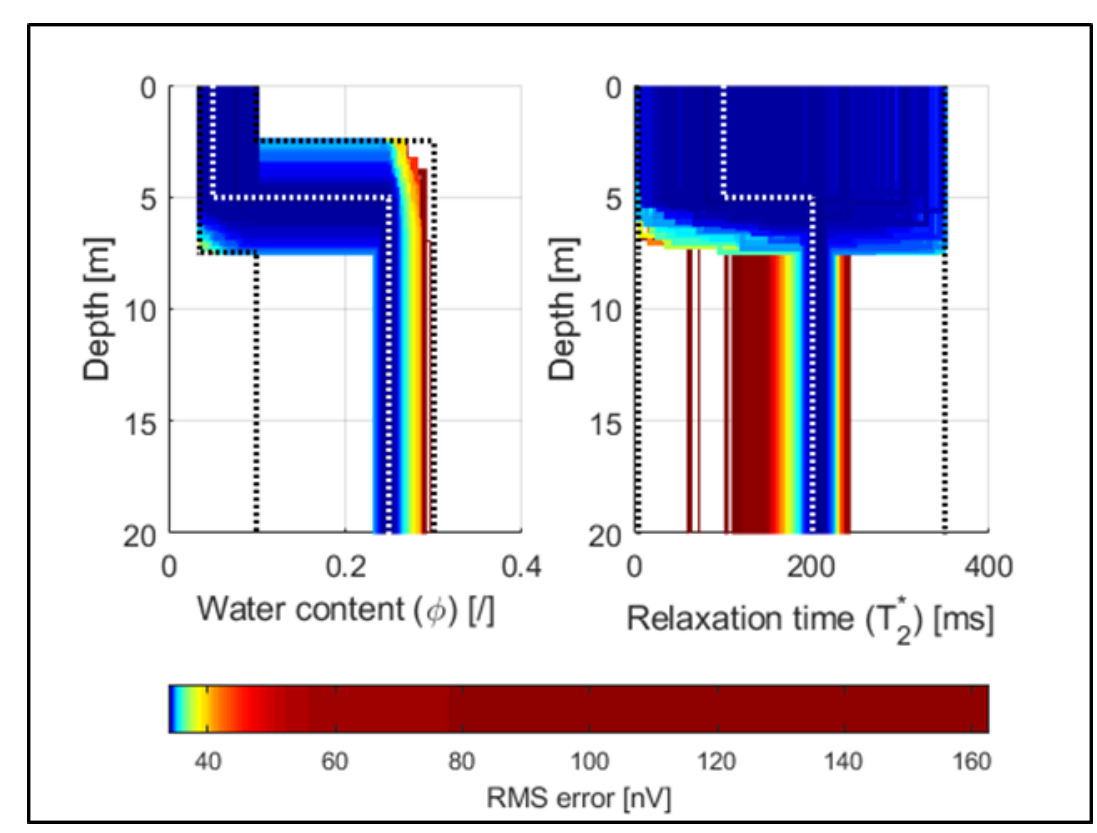




\section{BEL1D \\ Improving with IPR, the concept}

- IPR = Iterative Prior Resampling

- Inspired by Iterative Spatial Resampling (Mariethoz et al., 2010, Water Resour. Res.) and Sampling Importance Resampling (Dosne et al., 2016, J PHARMACOKINET PHAR)

- Process:

- Adding the sampled models to the prior

- Re-running the BEL1D operations

- Repeat until convergence:

- Threshold on the difference between the obtained distributions (Wasserstein distance in normalized space)
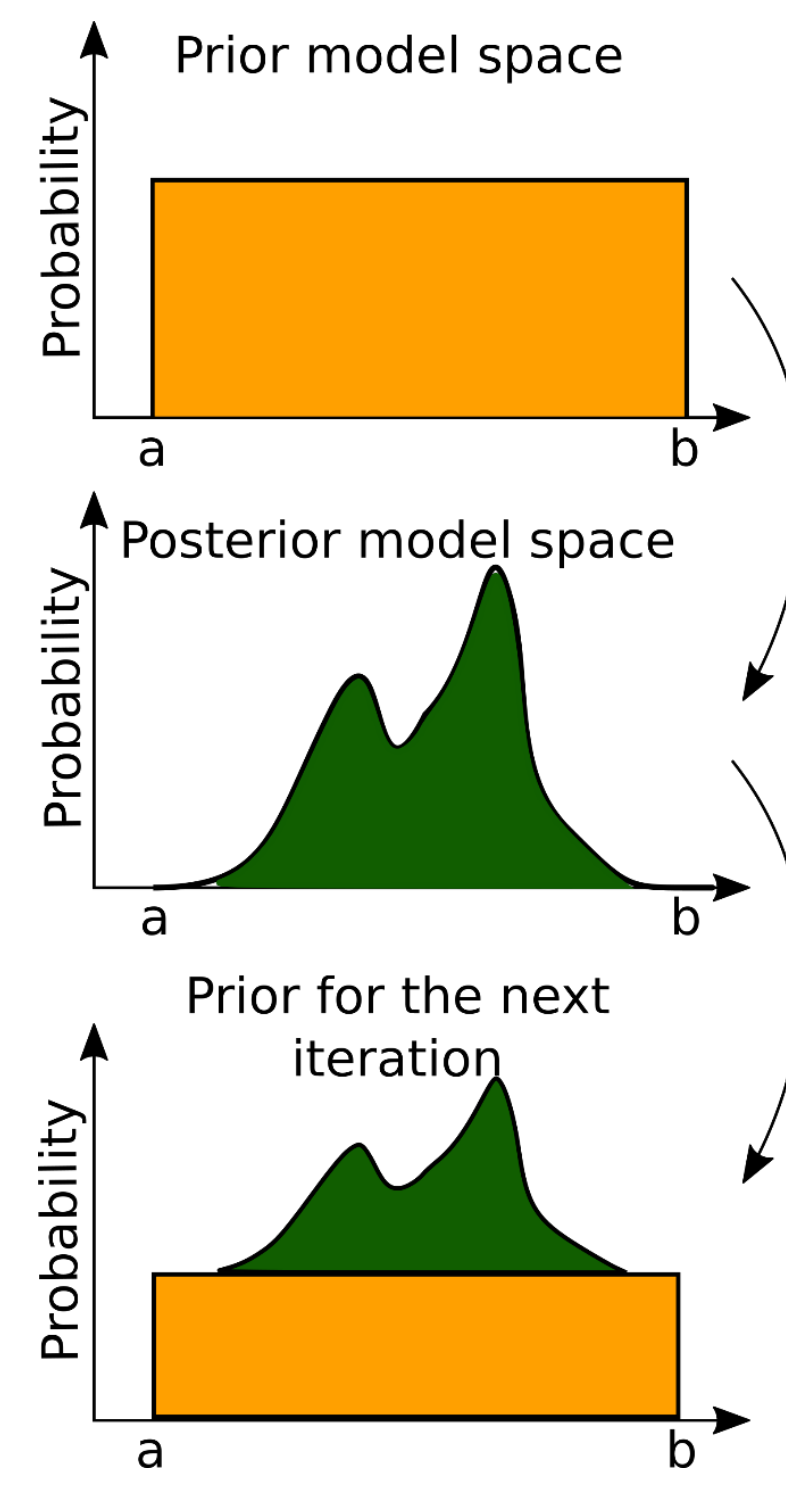


\section{Numerical benchmark}

- Simple 3-layer model

- Experimental design:

- Same transmitter/receiver loop

- 50 m diameter $\rightarrow$ penetration depth about 50 meters

- Noise $=10 n V$ (Gaussian)

- Prior defined accordingly but still large

\begin{tabular}{|c|c|c|c|c|c|c|c|c|c|}
\hline \multirow{2}{*}{ Layer \# } & \multicolumn{3}{|c|}{ Thickness e [m] } & \multicolumn{3}{c|}{ Water content W [\%] } & \multicolumn{3}{c|}{ Decay time $\mathbf{T}_{2}^{*}$ [ms] } \\
\hline & Min & True & Max & Min & True & Max & Min & True & Max \\
\hline $\mathbf{1}$ & 0 & 25 & 50 & 0 & 5 & 15 & 0 & 100 & 500 \\
\hline Half-space & 0 & 25 & 50 & 15 & 25 & 50 & 0 & 200 & 500 \\
\hline & 1 & Inf & $/$ & 0 & 10 & 15 & 0 & 50 & 500 \\
\hline
\end{tabular}




\section{Numerical benchmark Applying BEL1D}

Building the CCA space relationship
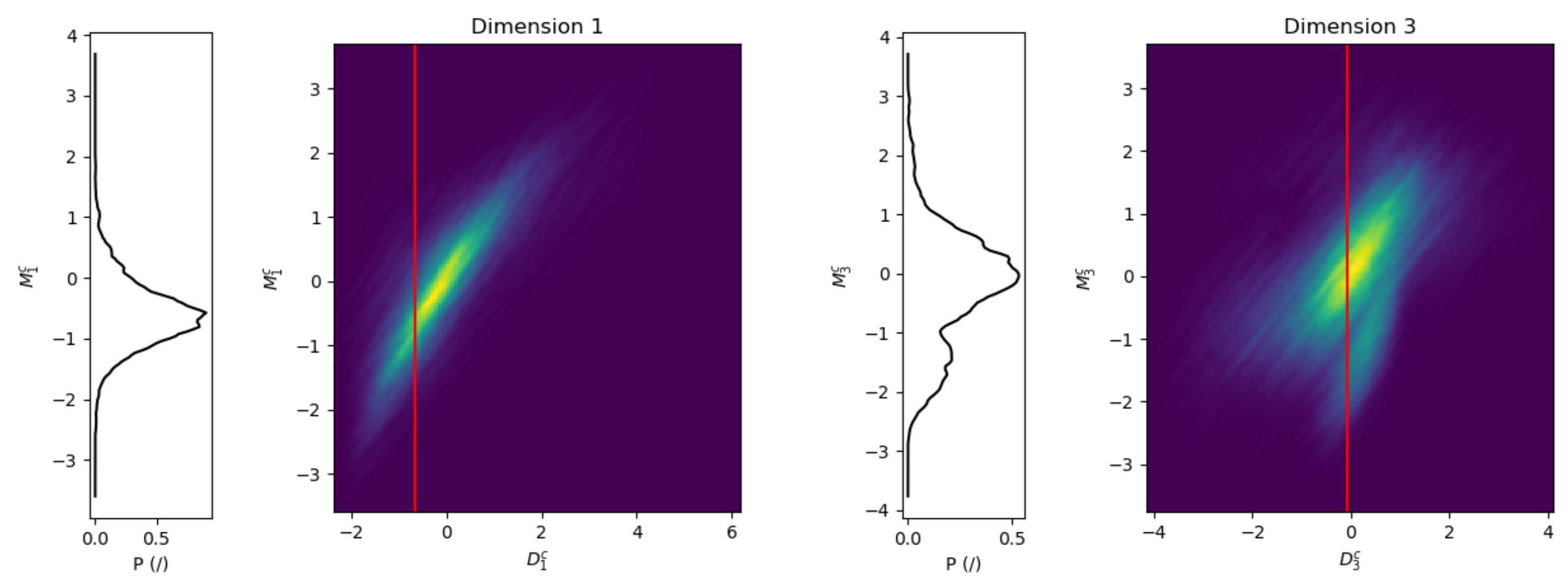


\section{Numerical benchmark}

Applying BEL1D

- Reduced uncertainty

- Still very large!

- RMSE above noise level

$\rightarrow$ Room for improvement

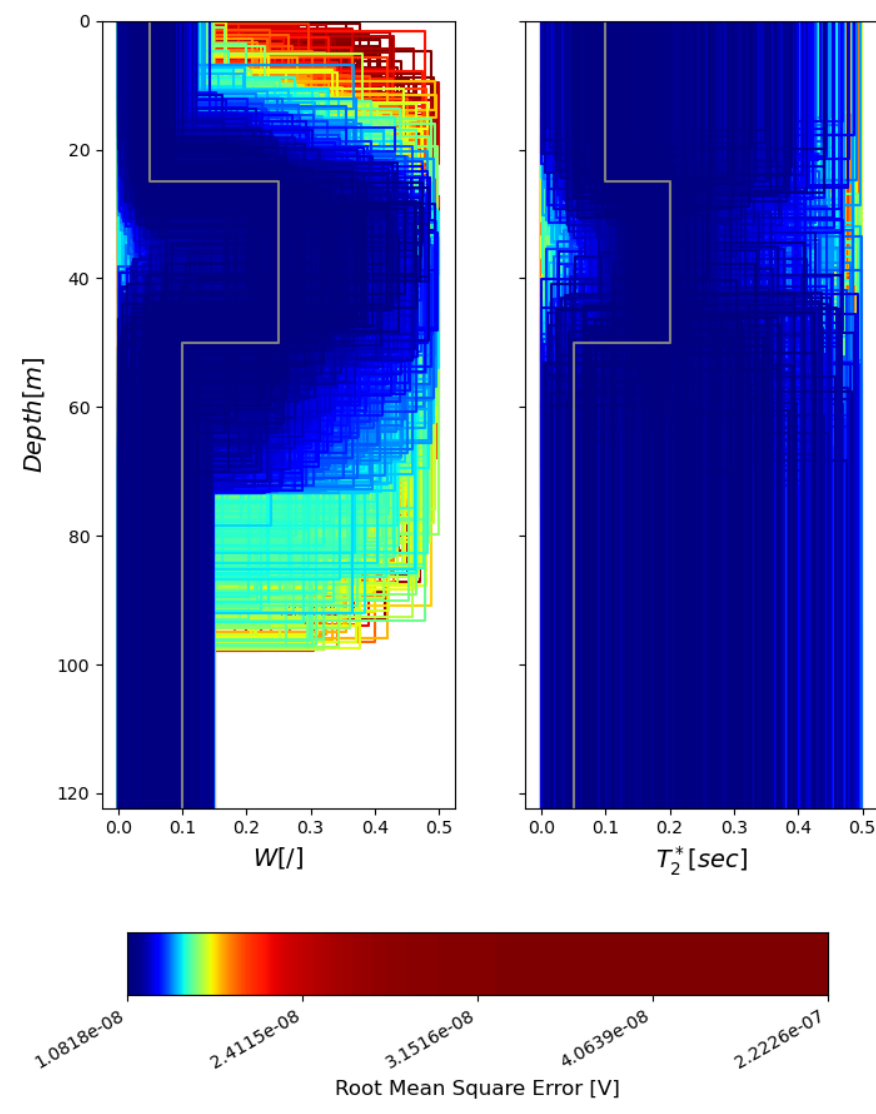

Improving BEL1D accuracy

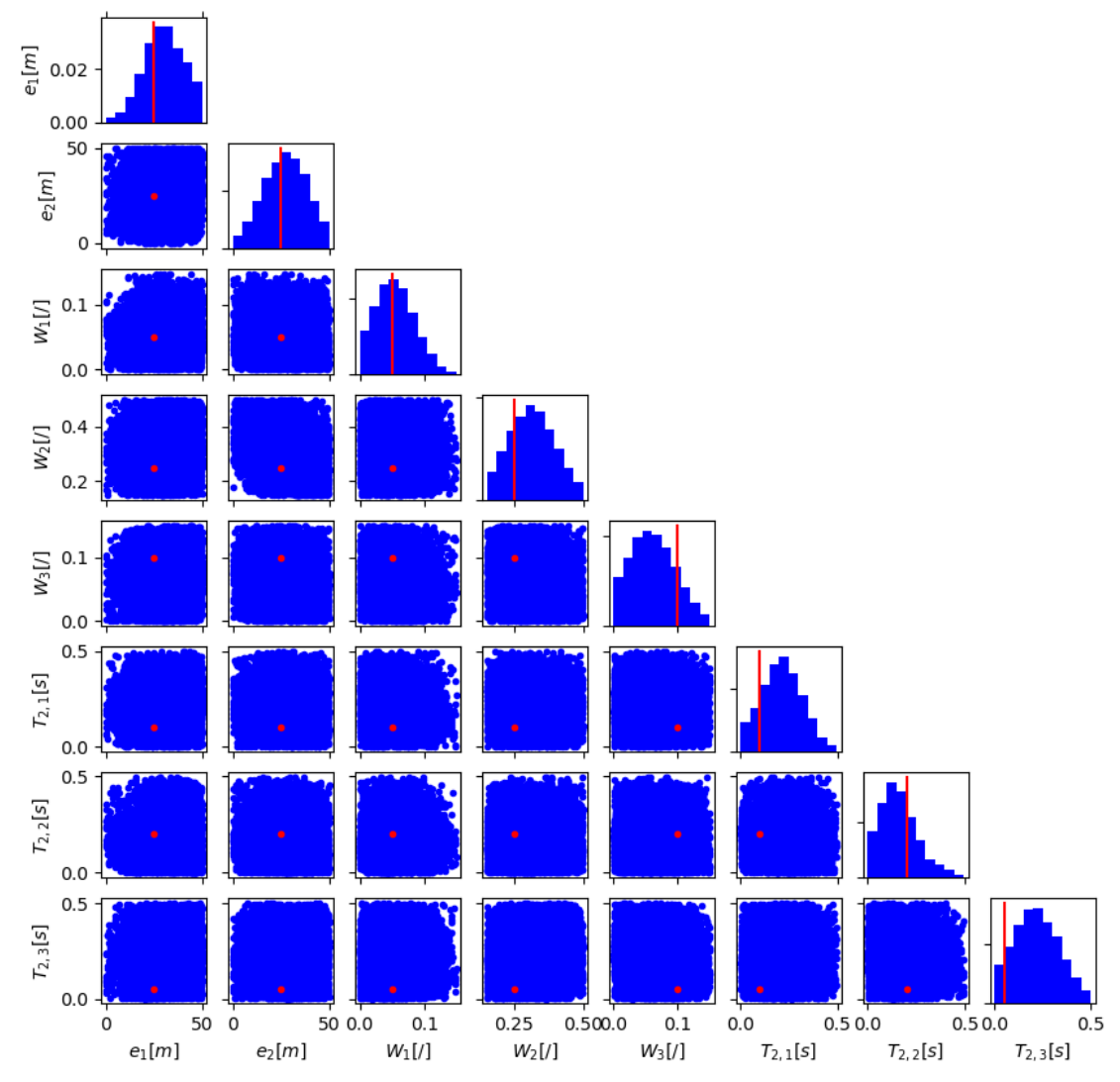

13

(SEG2O 


\section{Numerical benchmark Improving with IPR}

1st iteration

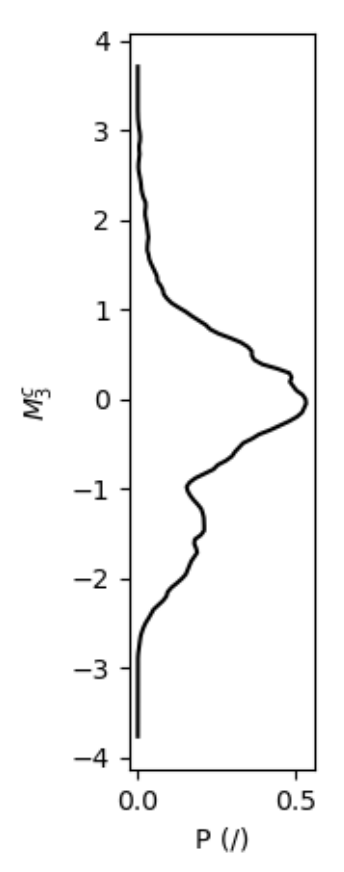

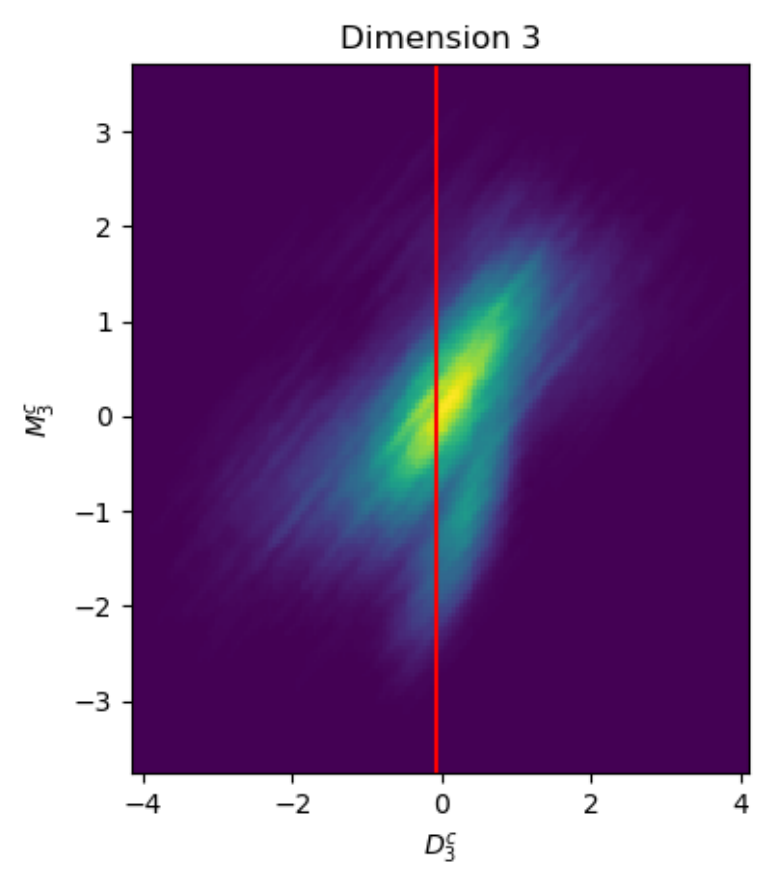

\section{Last iteration}
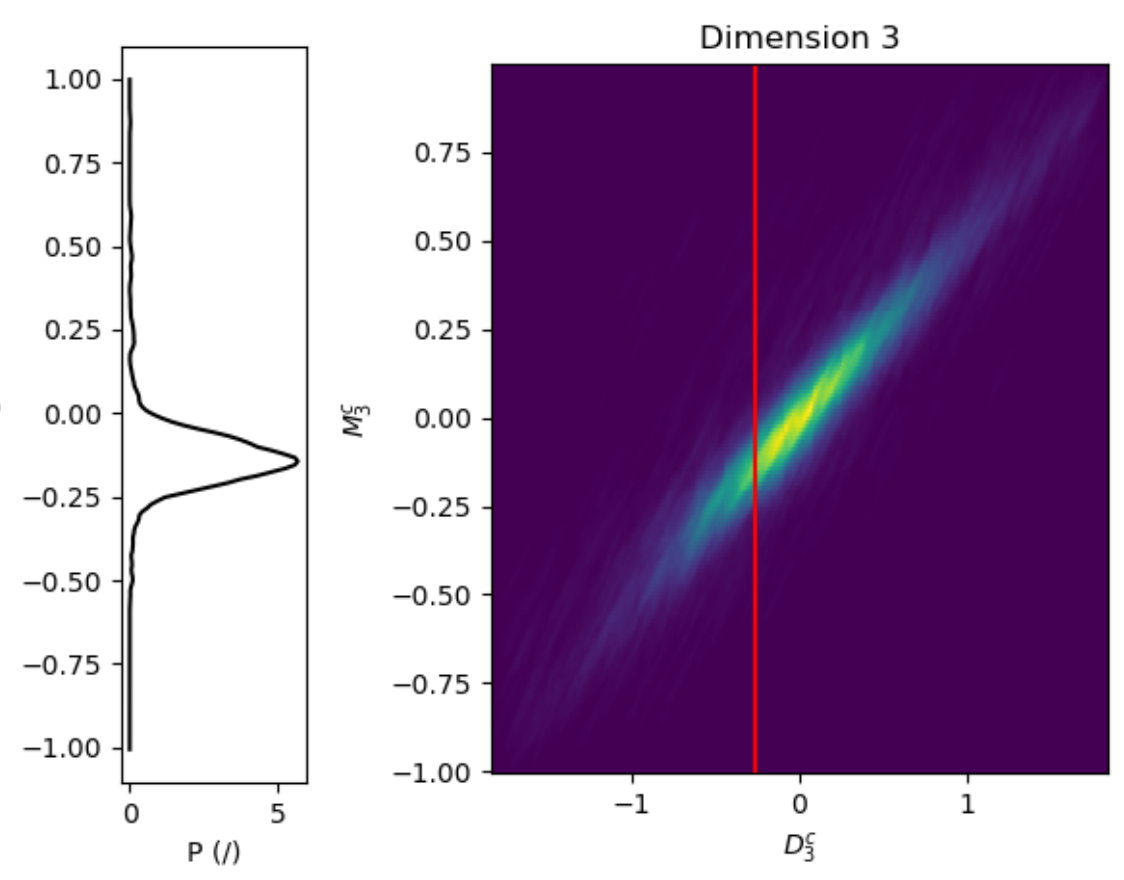

Improving BEL1D accuracy 


\section{Numerical benchmark}
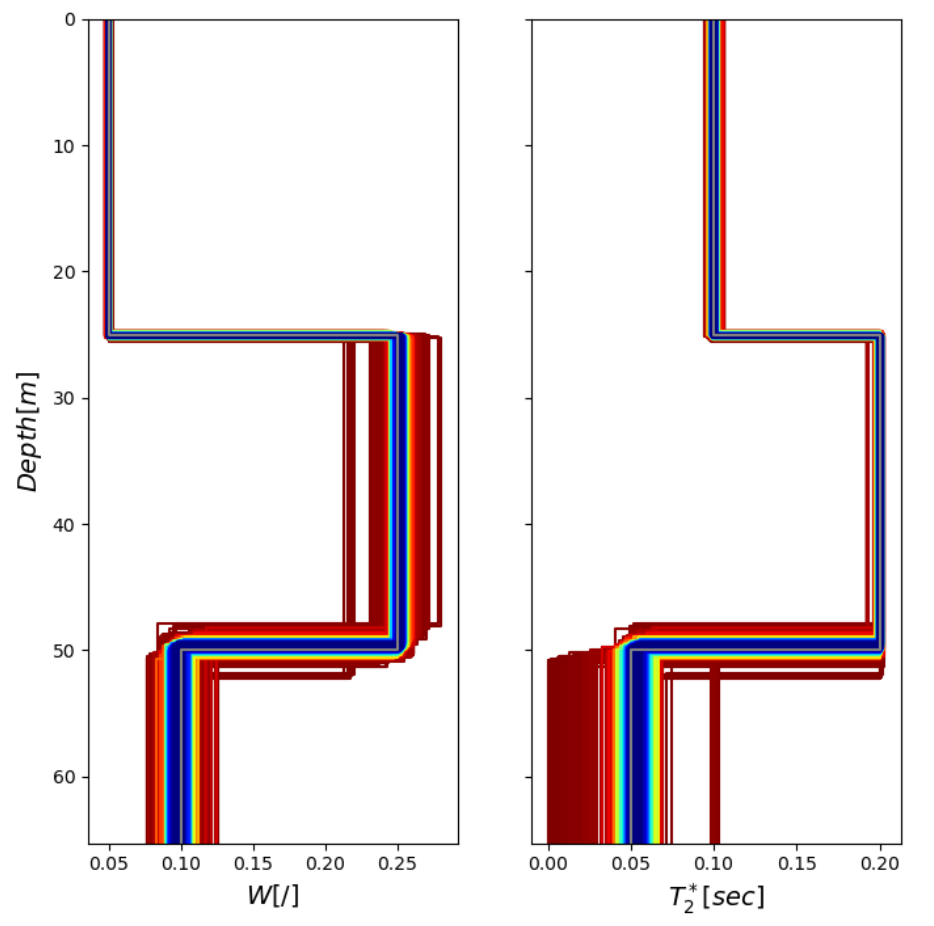

- Narrow uncertainty

- Sensitivity lower in depth

- From experimental design

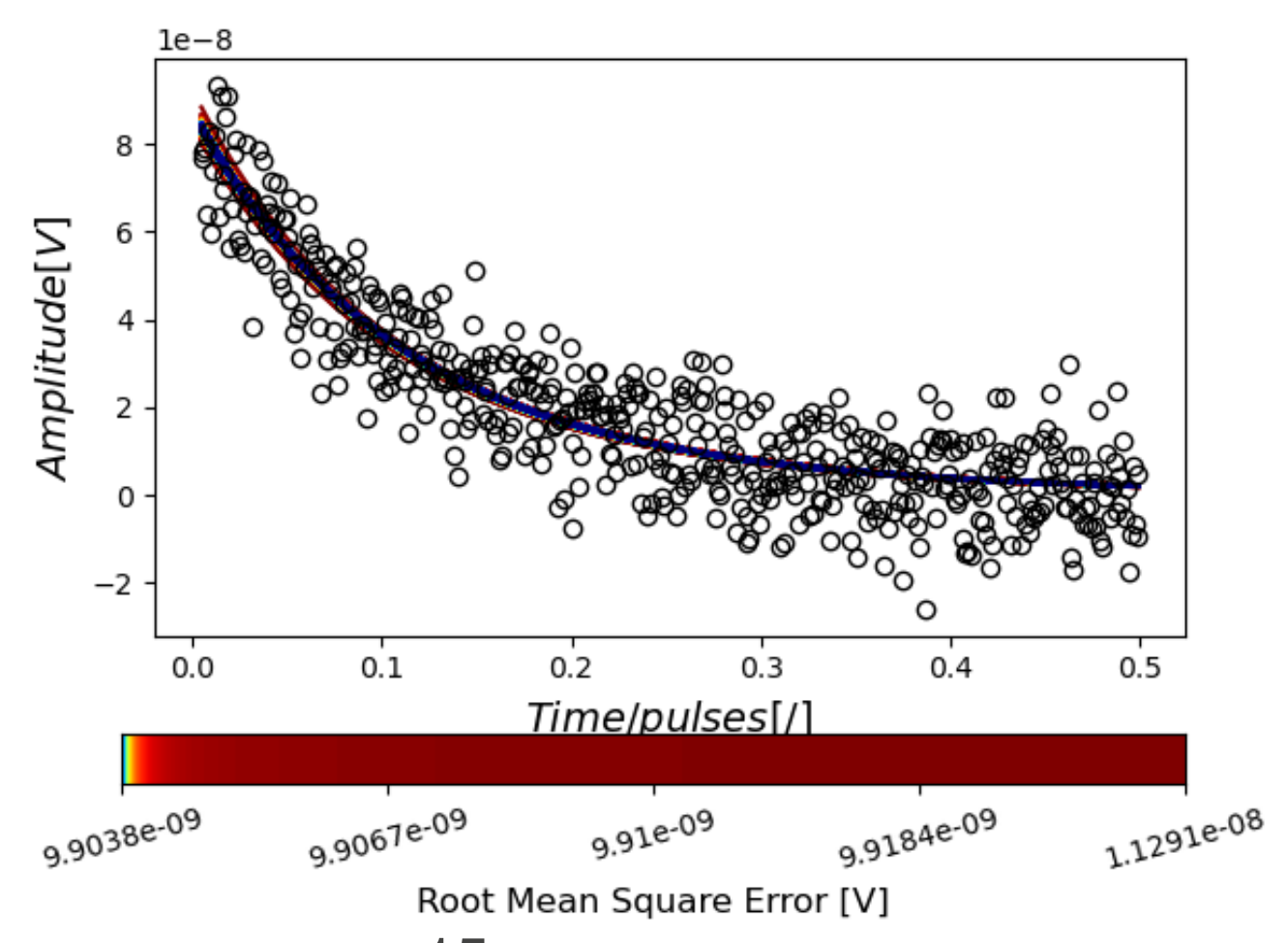




\section{Numerical benchmark}

Comparing with McMC

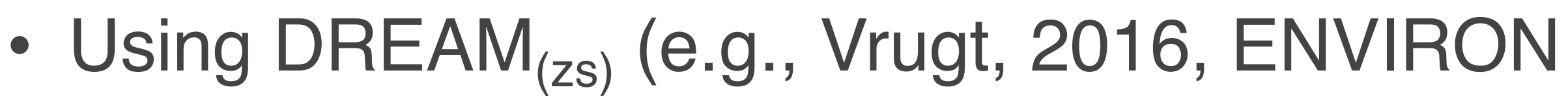
MODELL SOFTW and Laloy et al., 2018, Water Resour. Res.)

- Tuned to convergence:

- Number sequences: 20

- Samples per chain: 10,000

- Jump rate: 0.1 


\section{Numerical benchmark Comparing with McMC}

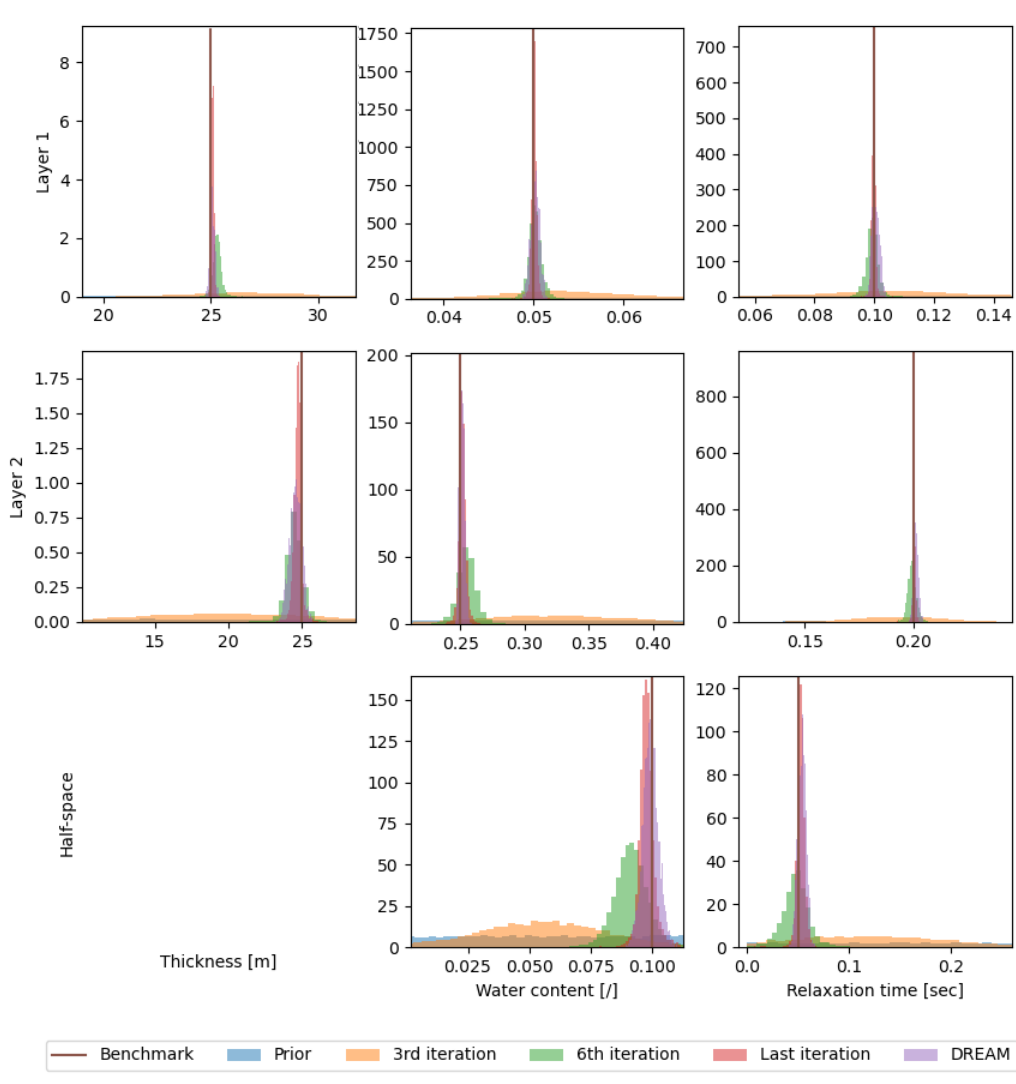

- The last iteration coincide with results from DREAM

- However:

- CPU time is lower (250 seconds for BEL1D vs 500 for $\operatorname{DREAM}_{(\mathrm{zs})}$ )

- Difficulty to tune to convergence in DREAM 


\section{Case study: Mont Rigi}

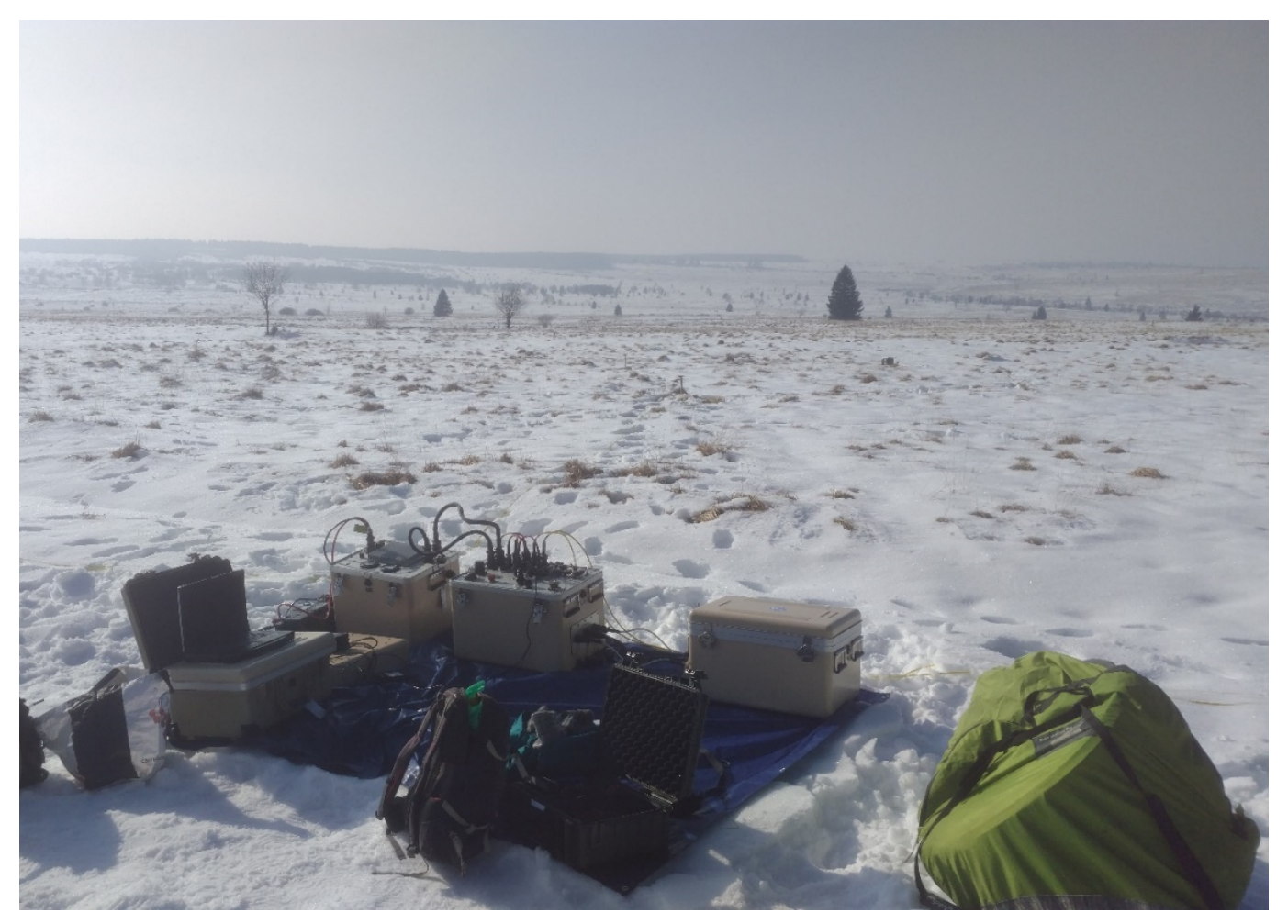

- Natural reserve in the Eastern part of Belgium

- Metric peat above Cambrian bedrock

- Experiment:

- Single transmitter/receiver

- 20 meters in diameter

- Noise $18 \mathrm{nV}$ 


\section{Case study: Mont Rigi}

First iteration
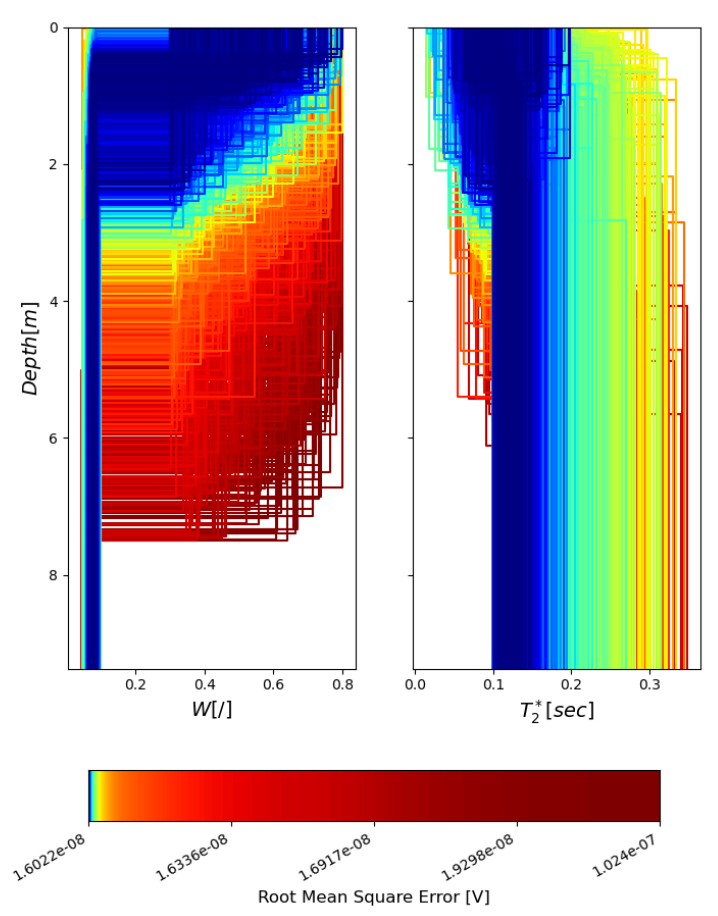

- Significant reduction of uncertainty

- Still an observable $\left(W_{1}, e_{1}\right)$ link

- Trend for the relaxation time

- CPU time $=30 \mathrm{sec}$
Last (6th) iteration

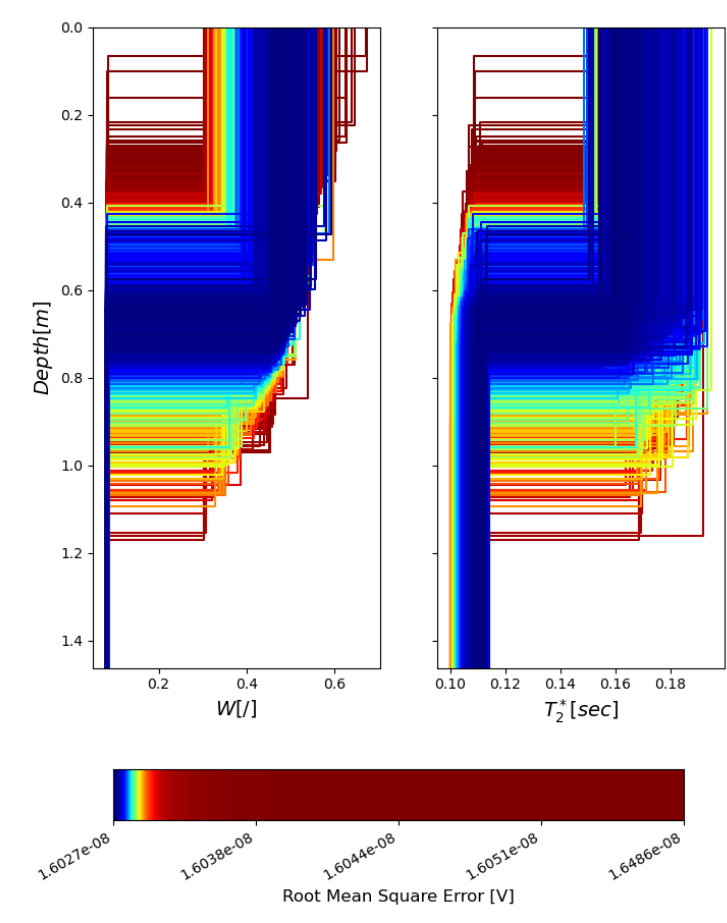




\section{Conclusion and perspectives}

- BEL1D combined with IPR is:

- Accurate (comparison with McMC)

- Efficient (CPU time)

- Easy to tune to convergence

- Significant improvement over BEL1D without iterations

- Currently developing other use case (MASW, EM)

- Near future: Smooth models, 2D, etc. 


\section{Conclusion and perspectives}

2D case - Time-Lapse ERT

- Time-lapse ERT (Hermans et al., 2016, Water Resour. Res.)

- Heat tracer experiment

- Captures the behavior of the heat tracer.
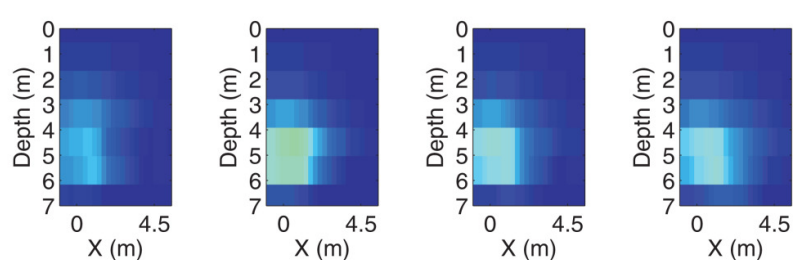

$\mathrm{t}=36 \mathrm{~h}$
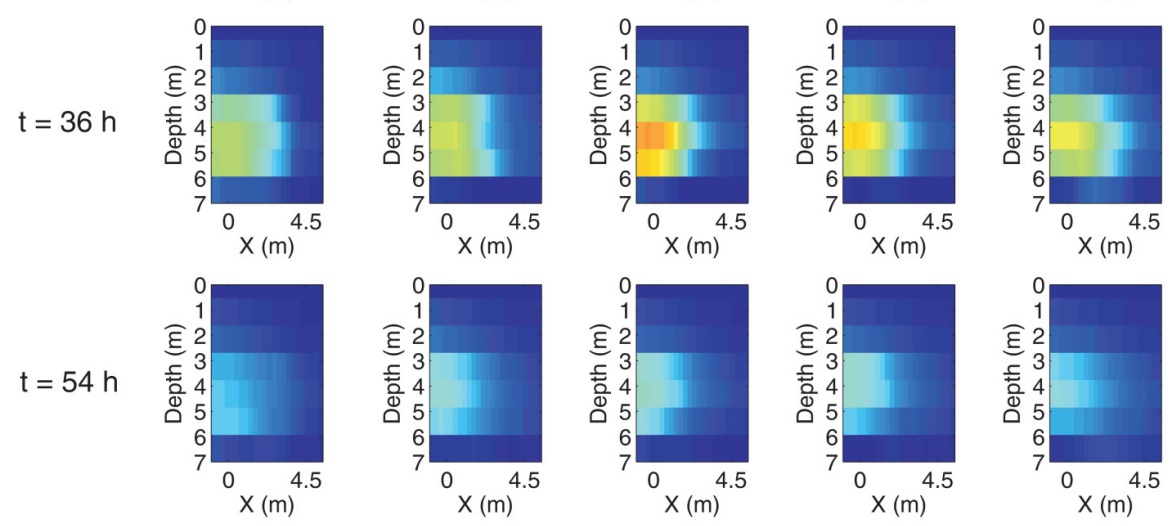

$t=72 h$

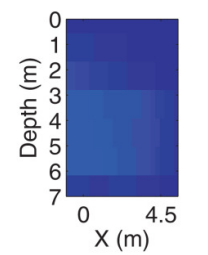

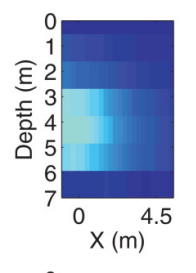
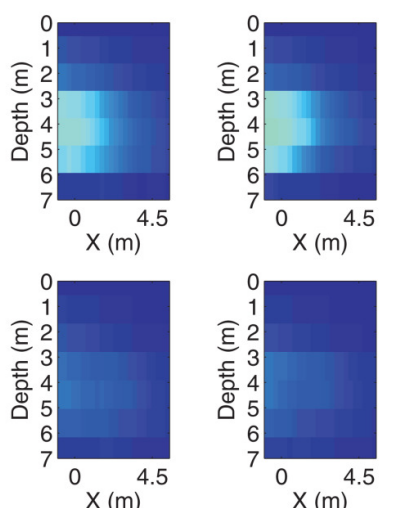
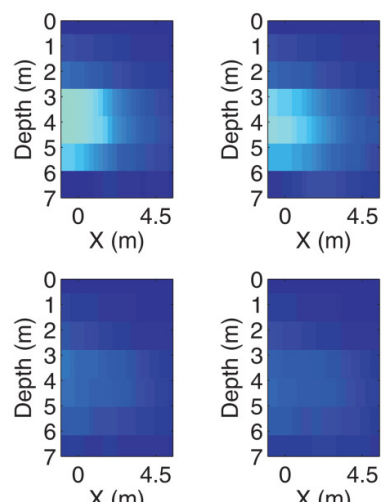


\section{Conclusion and perspectives Work in progress - Surface waves preview}

- Mirandola (Italy) case study from INTERPacific (Garofalo et al., 2016, SOIL DYN EARTHQ ENG)

- Comparison of the results with IPR with the different experts curves
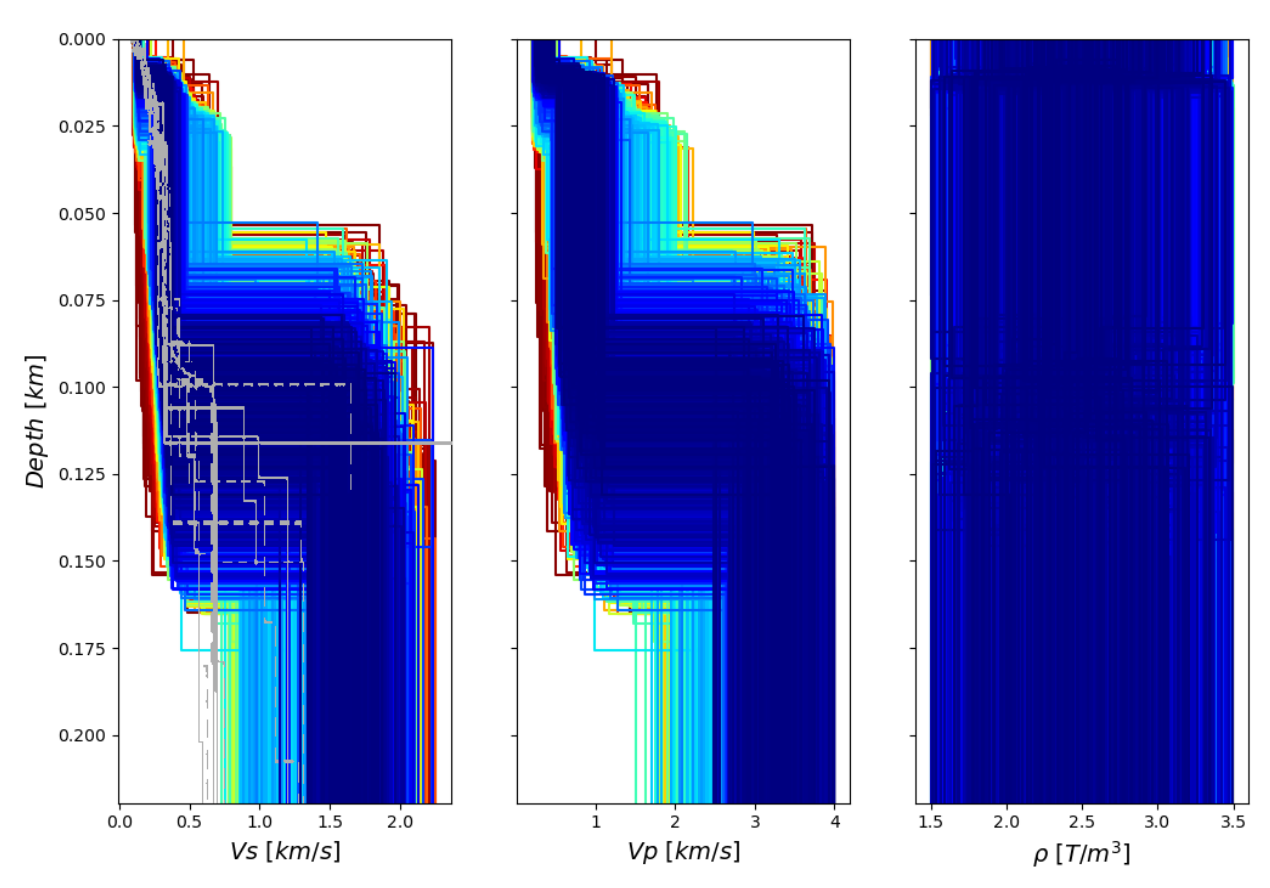


\section{Conclusion and perspectives Work in progress - Surface waves preview}

- Efficient reduction of the dataspace from the prior (gray) to the posterior at the last $\left(15^{\text {th }}\right)$ iteration

- The error model fits nicely the posterior dataspace

- The noise is not of the same kind as the one in SNMR

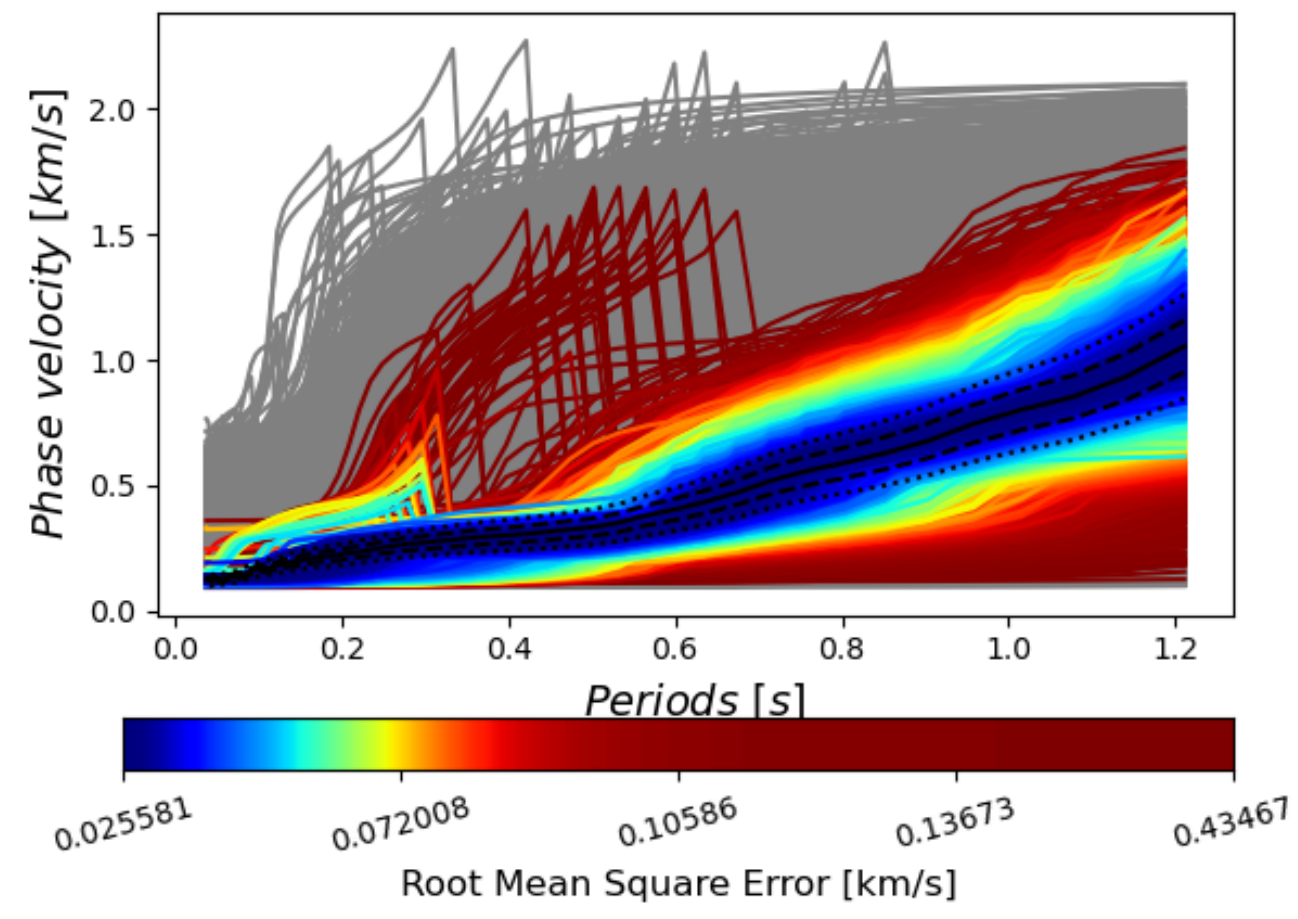




\section{Conclusion and perspectives Work in progress - Surface waves preview}

- Field benchmark available: Depth to the bedrock $=118 \mathrm{~m}$ (Garofalo et al., 2016, SOIL DYN EARTHQ ENG)

- Accurately reproduced by BEL1D

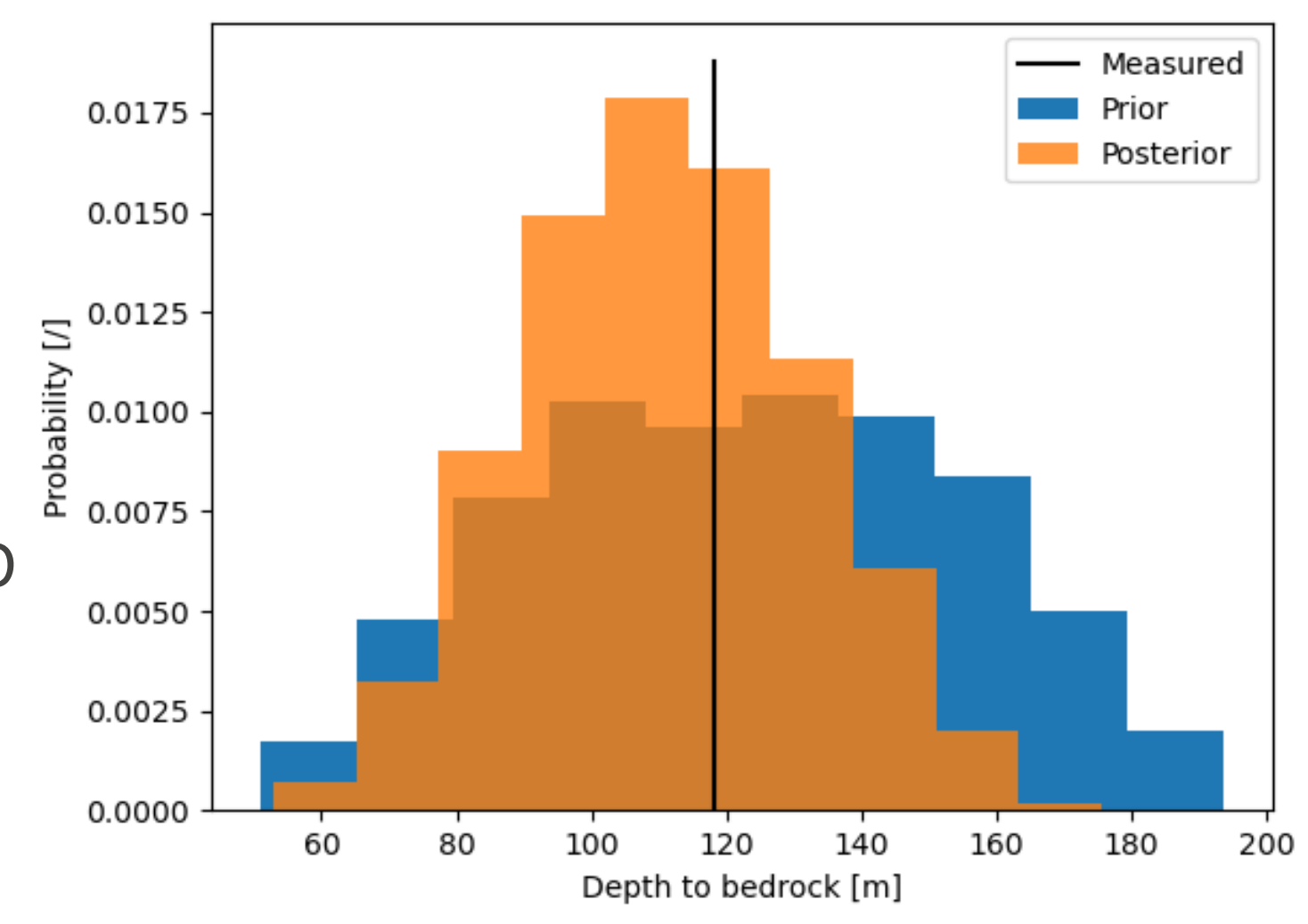




\section{Publication}

Computers \& Geosciences 138 (2020) 104456

Contents lists available at ScienceDirect

Computers and Geosciences

journal homepage: www.elsevier.com/locate/cageo

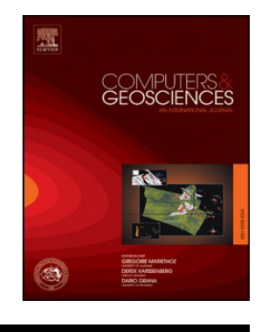

(D)

1D geological imaging of the subsurface from geophysical data with Bayesian Evidential Learning

Hadrien Michel ${ }^{a, b, c, *}$, Frédéric Nguyen ${ }^{a, d}$, Thomas Kremer ${ }^{a}$, Ann Elen ${ }^{d}$, Thomas Hermans ${ }^{b}$

${ }^{a}$ University of Liège, Urban and Environmental Engineering Department, Faculty of Applied Sciences, Liège, Belgium

${ }^{b}$ Ghent University, Department of Geology, Ghent, Belgium

c F.R.S.FNRS (Fonds de la Recherche Scientifique), Brussels, Belgium

${ }^{\mathrm{K} U} \mathrm{~L}$ Leuven, Department of Earth and Environmental Sciences, Leuven, Belgium

https://doi.org/10.1016/j.cageo.2020.104456 


\section{Thanks for your attention!}

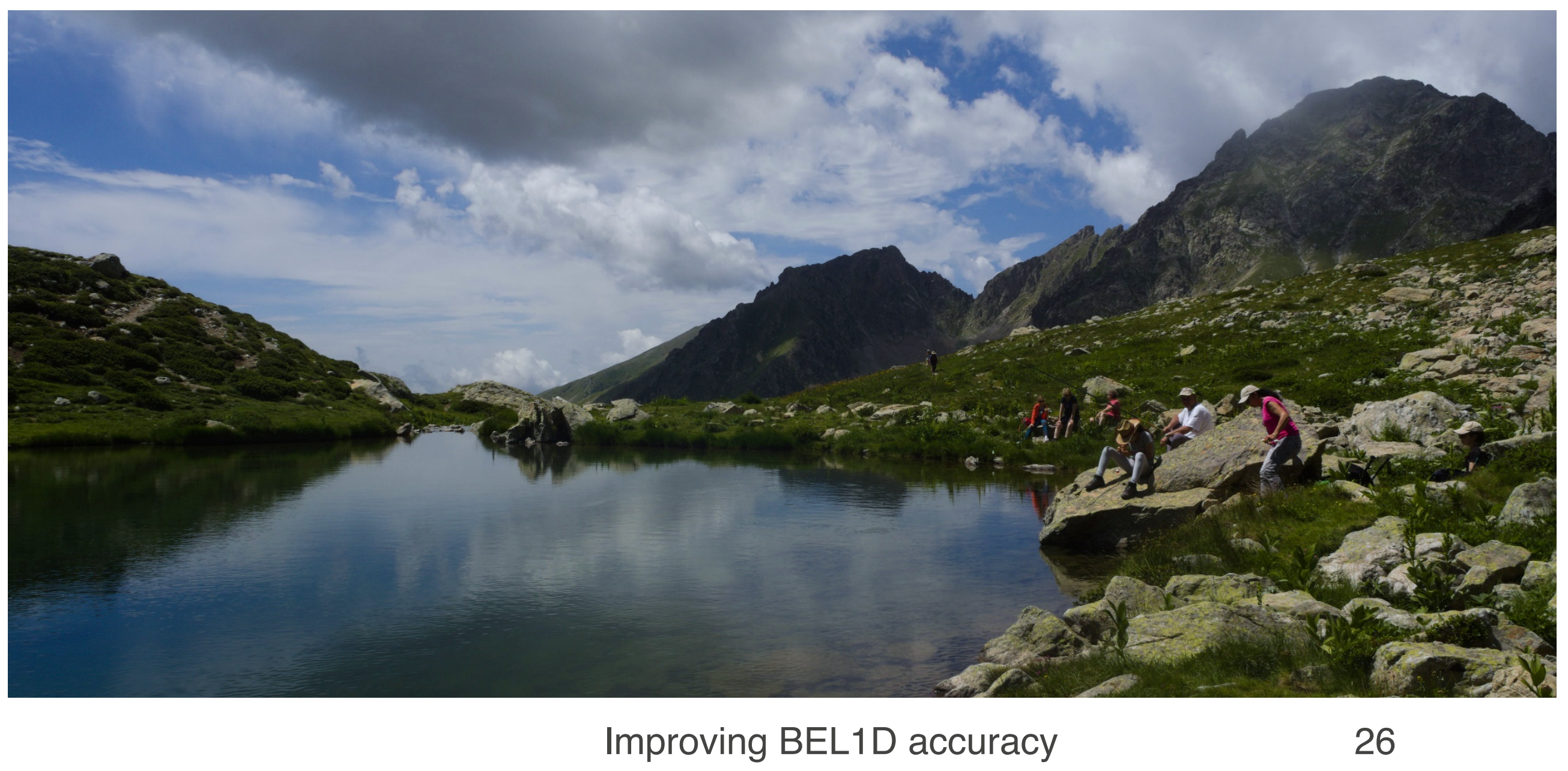

SEG20 


\section{Conclusion and perspectives}

- BEL1D combined with IPR is:

- Accurate (comparison with McMC)

- Efficient (CPU time)

- Easy to tune to convergence

- Significant improvement over BEL1D without iterations

- Currently developing other use case (MASW, EM)

- Near future: Smooth models, 2D, etc. 